\title{
Solutions of Ternary Problems of Conditional Probability with Applications to Mathematical Epidemiology and the COVID-19 Pandemic
}

\author{
Ali Muhammad Ali Rushdi \\ Department of Electrical and Computer Engineering, \\ Faculty of Engineering, King Abdulaziz University, \\ P. O. Box 80204, Jeddah, 21589, Saudi Arabia. \\ Corresponding author: arushdi@kau.edu.sa \\ Hamzah Abdul Majid Serag \\ Department of Electrical and Computer Engineering, \\ Faculty of Engineering, King Abdulaziz University, \\ P. O. Box 80204, Jeddah, 21589, Saudi Arabia. \\ E-mail: hlmajeedsiraj@stu.kau.edu.sa
}

(Received May 7, 2020; Accepted May 28, 2020)

\begin{abstract}
A normalized version of the ubiquitous two-by-two contingency matrix is associated with a variety of marginal, conjunctive, and conditional probabilities that serve as appropriate indicators in diagnostic testing. If this matrix is enhanced by being interpreted as a probabilistic Universe of Discourse, it still suffers from two inter-related shortcomings, arising from lack of length/area proportionality and a potential misconception concerning a false assumption of independence between the two underlying events. This paper remedies these two shortcomings by modifying this matrix into a new Karnaugh-map-like diagram that resembles an eikosogram. Furthermore, the paper suggests the use of a pair of functionally complementary versions of this diagram to handle any ternary problem of conditional probability. The two diagrams split the unknowns and equations between themselves in a fashion that allows the use of a divide-and-conquer strategy to handle such a problem. The method of solution is demonstrated via four examples, in which the solution might be arithmetic or algebraic, and independently might be numerical or symbolic. In particular, we provide a symbolic arithmetic derivation of the well-known formulas that express the predictive values in terms of prevalence, sensitivity and specificity. Moreover, we prove a virtually unknown interdependence among the two predictive values, sensitivity, and specificity. In fact, we employ a method of symbolic algebraic derivation to express any one of these four indicators in terms of the other three. The contribution of this paper to the diagnostic testing aspects of mathematical epidemiology culminates in a timely application to the estimation of the true prevalence of the contemporary world-wide COVID-19 pandemic. It turns out that this estimation is hindered more by the lack of global testing world-wide rather than by the unavoidable imperfection of the available testing methods.
\end{abstract}

Keywords- Ternary problem, Conditional probability, Length/area proportionality, Probabilistic dependence, Divide and conquer, Imperfect testing, Mathematical epidemiology, COVID-19.

\section{Introduction}

A ubiquitous tool of scientific analysis and research is the two-by-two contingency matrix (known also by a variety of other names such as the confusion table, the frequency matrix, the agreement table, or (even as) a truth table). This table arises in many diverse applications such as clinical testing, criminal investigations, judicial trials, lie detection, null-hypothesis acceptance/rejection, recommender systems, quality control, industrial management, satellite mapping, text and pattern classification, signal communications, DNA identification, forensic reasoning and machine classification (Amin et al., 2019; Rushdi and Talmees, 2019). Despite the 
International Journal of Mathematical, Engineering and Management Sciences

Vol. 5, No. 5, 787-811, 2020

https://doi.org/10.33889/IJMEMS.2020.5.5.062

inherent simplicity of this matrix, it is the source of a surprisingly huge number of indicators and somewhat complex relations. Its mathematical analysis has been the subject of many papers and books hitherto (see, e.g. Fienberg and Gilbert, 1970; Good and Mittal, 1987; Anderson and Finn, 1996; Johnson, 1999; Fawcett, 2006; Lesaffre et al., 2007; Parikh et al., 2008; Powers, 2011; Shindo et al., 2012; Johnson and Johnson, 2014; Costello and Watts, 2016; Kent and Hancock, 2016; Johnson, 2017). A notable attempt to simplify the analysis of a contingency matrix is to employ a normalized version of it, in which both its four entries and four indexes are interpreted as probabilities (Rushdi and Rushdi, 2018b, 2019), thereby reducing the analysis of this matrix to a ternary problem of conditional probability, which is a problem of probability involving some conditional probability, with exactly three given quantities (Carles and Huerta, 2007; Cerdán and Huerta, 2007; Edo et al., 2011; Huerta, 2009, 2014; Huerta, et al., 2011; Rushdi et al., 2018; Rushdi and Rushdi, 2019).

This paper is a further improvement of the aforementioned normalized contingency matrix, which converts it into a length/area-proportional diagram that explicitly shows whether the two underlying events are mutually supporting (favoring), mutually independent, or mutually weakening (disfavoring). Two different (albeit functionally complementary) versions of this diagram are used in unison through their sharing of the same four entries, which represent the four relevant conjunctive probabilities. In contrast to the conventional normalized matrix, which has just four indexes representing the four pertinent marginal probabilities, our two novel diagrams have six indexes each, allowing them together to explicitly represent not only these four marginal probabilities, but the eight concerned conditional probabilities as well. The combined employment of these two novel diagrams constitutes a divide-and-conquer methodology for handling a ternary problem of conditional probability, as such an employment allows the splitting of the unknowns and equations almost evenly (with a minimal overlapping) between the two diagrams.

The organization of the rest of this paper is as follows. Section 2 is a brief primer about conditional probability and the nature of inter-dependence (or lack thereof) between two probabilistic events. Section 3 is a detailed exposition of the normalized two-by-two contingency table and the eight most prominent indicators of diagnostic testing, which are typically used in association with this table. Section 4 introduces two length/area-proportional variants of this normalized matrix, each of which provides its own explicit visualization of event interdependence. These two variants become identical if the underlying events are independent. Section 5 introduces and studies the nature of ternary problems of conditional probability. Section 6 discusses how the new length/area-proportional variants of the normalized contingency matrix can be used to solve ternary problems of conditional probability. Section 6 demonstrates the method of solution via four examples. Examples 1 and 2 obtain numerical solutions via arithmetic and algebraic techniques, respectively. Example 3 provides a symbolic arithmetic derivation of the well-known formulas that express the predictive values in terms of prevalence, sensitivity and specificity. Example 3 also provides other prevalence-related formulas. Example 4 proves a virtually unknown inter-dependence among the two predictive values, sensitivity, and specificity. In fact, Example 4 utilizes a method of symbolic algebraic derivation to express any one of these four indicators in terms of the other three. As a bonus, Section 7 augments our exploration of the diagnostic testing aspects of mathematical epidemiology by a timely application to and a discussion of the contemporary world-wide COVID-19 pandemic. Section 8 concludes the paper. 
International Journal of Mathematical, Engineering and Management Sciences

Vol. 5, No. 5, 787-811, 2020

https://doi.org/10.33889/IJMEMS.2020.5.5.062

\section{On Conditional Probability}

Despite disagreement on the meaning of probability, there is some consensus on its basic axioms (Da Costa, 1986). Herein, we employ the empirical (frequentistic or common-sense) interpretation of probability, and base its concepts on a probability "Universe of Discourse" or "sample space" which constitutes the set of all possible (equally-likely) outcomes or primitive indecomposable events of an underlying conceptual random "experiment." We define events as subsets of the sample space, and hence get $\mathrm{N}=2^{n}$ events for a sample space of $n$ outcomes or sample points (Rushdi and Rushdi, 2018b; 2019). Since events are sets, we can also describe events via elementary set operations (complementation, intersection, union, and set difference). Since the outcomes in a sample space are equally likely, the probability $P(A)$ of an event $A$ is defined as the number of outcomes constituting $A$ (favoring $A$ ) divided by the total number of outcomes in the sample space. The concept of conditional probability $P(A \mid B)$ of an event $A$ given an event $B$ is based on the notion that conditioning on an event $B$ of non-zero probability means that $B$ replaces the universal set $S$ as a certain event (Rushdi and Rushdi, 2017). The orthodox way of defining conditional probability (Hájek, 2003) is as the quotient (ratio) of the conjunctive probability $P(A \cap B)$ by the probability of the conditioning event $B$, provided this latter probability is not zero, namely

$P(A \mid B)=P(A \cap B) / P(B), \quad P(B) \neq 0$.

Rushdi and Rushdi (2018b; 2019) utilized the Venn diagram and the Karnaugh map to visualize a conditional probability as the ratio of two areas, provided the diagram and map be interpreted as area-proportional ones. We stress that a conditional probability is just a probability; it satisfies the axioms of probability and it is a dimensionless number of a real value that belongs to the closed unit interval $[0.0,1.0]$. An Unconditional probability is simply a conditional one, in which conditioning is with respect to the certain event.

There are three different ways in which the occurrence of an event $B$ affects or influences the occurrence of an event $A$ (Falk and Bar-Hillel, 1983; Krämer and Gigerenzer, 2005; Rushdi and Rushdi, 2019). These are:

1- Positive dependence: $B$ favors or supports $A$, i.e., the occurrence of $B$ increases the probability of occurrence of $A(P(A \mid B)>P(A))$, denoted $B>A$,

2- Independence: $B$ does not affect $A$, i.e., the occurrence of $B$ leaves the probability of occurrence of $A$ unchanged $(P(A \mid B)=P(A))$, denoted $B \perp A$,

3- Negative dependence: $B$ disfavors or weakens $A$, i.e., the occurrence of $B$ decreases the probability of occurrence of $A(P(A \mid B)<P(A))$, denoted $B \searrow A$.

The three relations $B \rtimes A, B \perp A$, and $B \searrow A$ are symmetric, and hence we should speak about mutual (in)dependence rather than about unilateral (in)dependence. None of the three relations is transitive. Generally, we note that the relations $B \perp A$, and $B \searrow A$ are not reflexive, while the relation $B>A$ is. The relation $B>A$ is equivalent to each of the relations $B \backslash \bar{A}$ and $\bar{B} \nearrow \bar{A}$. A common fallacy is to attribute to probabilistic support $\lambda$ (between events), the properties of the logical relationship of implication (between propositions), which is denoted $A \rightarrow B$ (Falk and Bar-Hillel, 1983; Rushdi and Rushdi, 2019). 
International Journal of Mathematical, Engineering and Management Sciences

Vol. 5, No. 5, 787-811, 2020

https://doi.org/10.33889/IJMEMS.2020.5.5.062

\section{The Normalized Two-by-Two Contingency Table}

A prominent and ubiquities domain in which conditional probabilities naturally arise is that of a normalized contingency matrix (Rushdi, et al., 2018; Rushdi and Rushdi, 2018b; 2019). Figure 1 demonstrates a two-by-two contingency matrix for metric or test $i$ with respect to metric or test $j$. Two dichotomous variables are involved, each of which is of a value belonging to the set $\{+1,-1\}$ of indices. The metric or test $i$ (typically a new test to be assessed) is reporting positive cases (of the value +1 ), in which the (usually adverse) attribute, trait, or condition is present, or reporting negative cases (of the value -1 ), in which this condition is absent. This test or metric is judged or evaluated by a reference or standard metric $j$ (typically a gold standard or the best test available), which has its own labeling of cases, again as positive or negative. If the reference metric $j$ agrees with the assessed metric $i$ then $j$ designates the case of $i$ as "true", and if $j$ disagrees with $i$ then the reference metric designates the case of the assessed one as "false." Therefore, the matrix four entries are called True Positives $\left(T P_{i j}\right)$, False Positives $\left(F P_{i j}\right)$, False Negatives $\left(F N_{i j}\right)$, and True Negatives $\left(T N_{i j}\right)$. The sum of these entries is the size of the reported population or total number of cases $\mathrm{N}$. The subscripts $i j$ assert the notion that $i$ is assessed or measured relative to $j$.

\begin{tabular}{|c|c|c|}
\hline$i$ & +1 & -1 \\
\hline+1 & $\begin{array}{c}T P_{i j} \\
\text { (True Positives) }\end{array}$ & $\begin{array}{c}F P_{i j} \\
\text { (False Positives) } \\
\text { (Type I Error) }\end{array}$ \\
\hline-1 & $\begin{array}{c}F N_{i j} \\
\text { (False Negatives) } \\
\text { (Type II Error) }\end{array}$ & $\begin{array}{c}T N_{i j} \\
\text { (True Negatives) }\end{array}$ \\
\hline
\end{tabular}

Figure 1. The two-by-two contingency matrix of test or metric $i$ with respect to test or metric $j$. This matrix has integer entries that add to the total number of cases $N$. (Typically, $j$ represents reality as a gold standard while $i$ is a measure to be assessed)

As Rushdi and Rushdi (2018a) point out, the afore-mentioned four elements of the standard contingency matrix represent non-normalized conjunctive probabilities. When we divide each of these entries by the total number of cases, we obtain the normalized contingency matrix in Figure 2 , whose entries are true conjunctive probabilities. Here, we use the symbols $A=\{j=+1\}$ and $B=\{i=+1\}$ to denote the events of positive cases (disease presence) according to the true or gold standard, and according to the new or assessed test, respectively. There are eight conditional 
International Journal of Mathematical, Engineering and Management Sciences

Vol. 5, No. 5, 787-811, 2020

https://doi.org/10.33889/IJMEMS.2020.5.5.062

probabilities concerning these two events and their complements, as shown in Figure 3. These can be identified as the eight most prominent measures or indicators used in diagnostic testing. These are the Sensitivity $\left(\operatorname{Sens}_{i j}\right)$ or True Positive Rate $\left(T P R_{i j}\right)$, the Specificity $\left(\operatorname{Spec}_{i j}\right)$ or True Negative Rate $\left(T N R_{i j}\right)$, the Positive and Negative Predictive Values $\left(P P V_{i j}\right.$ and $\left.N P V_{i j}\right)$, together with their respective complements (to 1.0), namely the False Negative Rate $\left(F N R_{i j}\right)$, False Positive Rate $\left(F P R_{i j}\right)$, False Discovery rate $\left(F D R_{i j}\right)$ and False Omission Rate $\left(F O R_{i j}\right)$ (Rushdi and Rushdi, 2018a, Rushdi et al., 2018; Rushdi and Talmees, 2018; 2019). The former four indicators are direct or agreement measures while the latter four serve as discrepancy or disagreement measures between the two tests or metrics $i$ and $j$. Due to the four complementation relations within pairs of these eight measures, the number of independent quantities among them is at most four. We show in Section 5 that this number is, in fact, three.

\begin{tabular}{|c|c|c|}
\hline & $P(A)=P(j=+1)$ & $P(\bar{A})=P(j=-1)$ \\
\hline $\begin{array}{c}P(B)= \\
P(i=+1)\end{array}$ & $P(A \cap B)=\frac{T P_{i j}}{N}$ & $P(\bar{A} \cap B)=\frac{F P_{i j}}{N}$ \\
\hline $\begin{array}{c}P(\bar{B})= \\
P(i=-1)\end{array}$ & $P(A \cap \bar{B})=\frac{F N_{i j}}{N}$ & $P(A \cap B)=\frac{T N_{i j}}{N}$ \\
\hline
\end{tabular}

Figure 2. The contingency matrix in Figure 1 normalized as a unit square representing a Universe of Discourse involving the two events $A$ and $B$ without area proportionality. The matrix has fractional nonnegative entries that add up to unity 


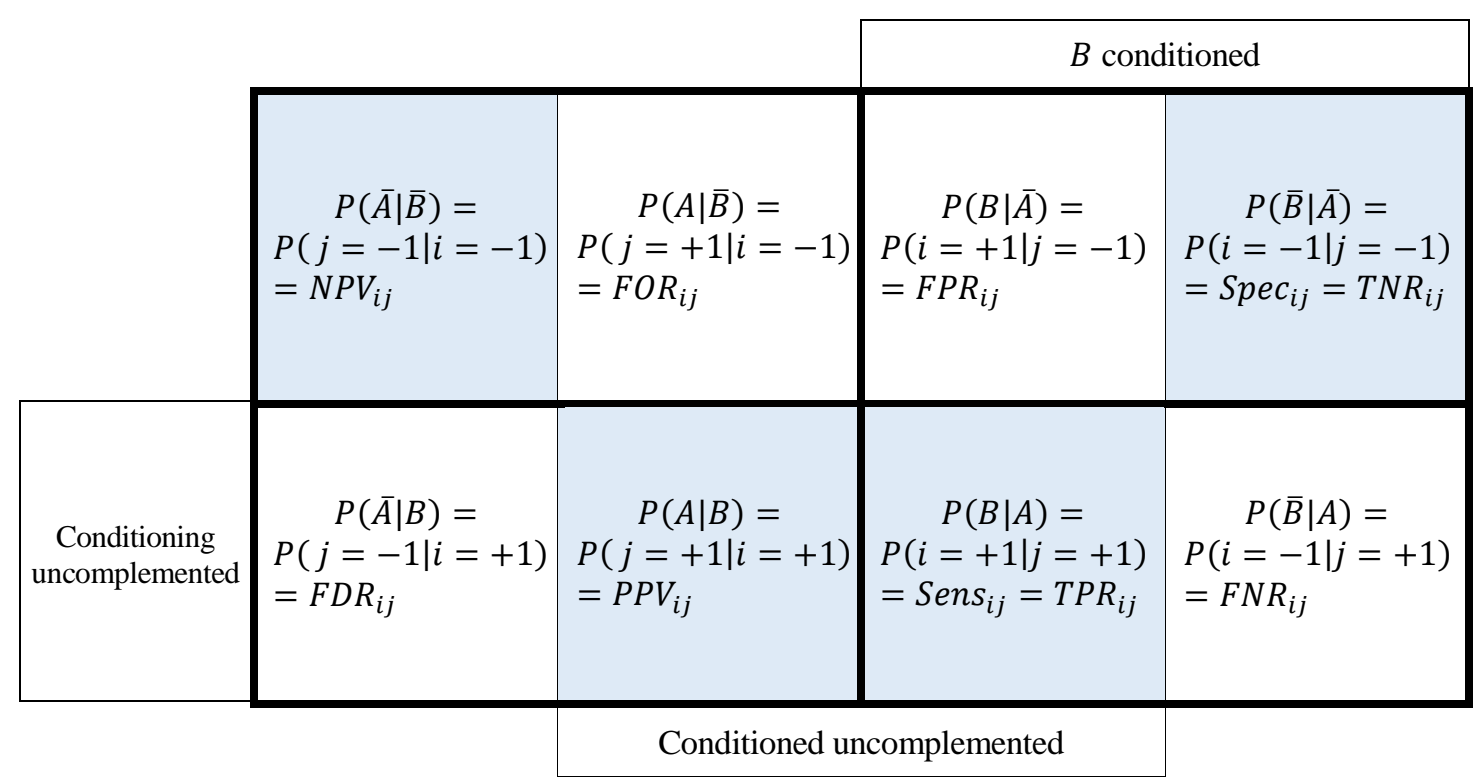

Figure 3. Definition of the eight conditional probabilities concerning events $A$ and $B$. The four most prominent among them (lacking mixed polarity) are entered in shaded cell. The graph has four major cells, each containing two complementary indicators

\section{The Length/Area-Proportional Normalized Table}

The normalized contingency matrix in Figure 2 might be thought to represent the Universe of Discourse or the probability Sample Space, i.e., it might be considered a Karnaugh map or a Venn diagram. However, it is not a length/area-proportional diagram, and its viewers might mistakenly assume that events $A$ and $B$ are necessarily independent if they inadvertently assume that the area allotted to a conjunctive event and the length assigned to a marginal event are proportional to their probabilities. To rule out such an assumption, we replace the Universe of Discourse of Figure 2 by one of two different length/area-proportional Venn diagrams shown in Figure 4, which are valid for positive inter-dependence between events $A$ and $B$. The simplest way to attain area-proportionality in Venn diagrams is to use rectilinear rather than curvilinear boundaries (Chow and Ruskey, 2003; Rushdi and Rushdi, 2018b; 2019), and hence the two diagrams are better (and henceforth will be) described as Karnaugh-map-like ones. An offshoot of using rectilinear rather than curvilinear boundaries is that length proportionality is additionally attained beside area proportionality. Each diagram is an obvious liaison among ten probabilities, namely, the four shared conjunctive probabilities (represented as proportional areas), two specific marginal probabilities of its own (represented as proportional lengths) and four specific conditional probabilities of its own (also represented as proportional lengths). The case of negative inter-dependence between events $A$ and $B$ might be handled in an analogous way (Figure 5 ), while in the case of independence between events $A$ and $B$, the two diagrams become the same single diagram, and all conditional probabilities become equal to marginal ones (Figure 6). Without loss of generality, we will consider only examples pertaining to Figure 4 in the rest of this paper. 
International Journal of Mathematical, Engineering and Management Sciences

Vol. 5, No. 5, 787-811, 2020

https://doi.org/10.33889/IJMEMS.2020.5.5.062
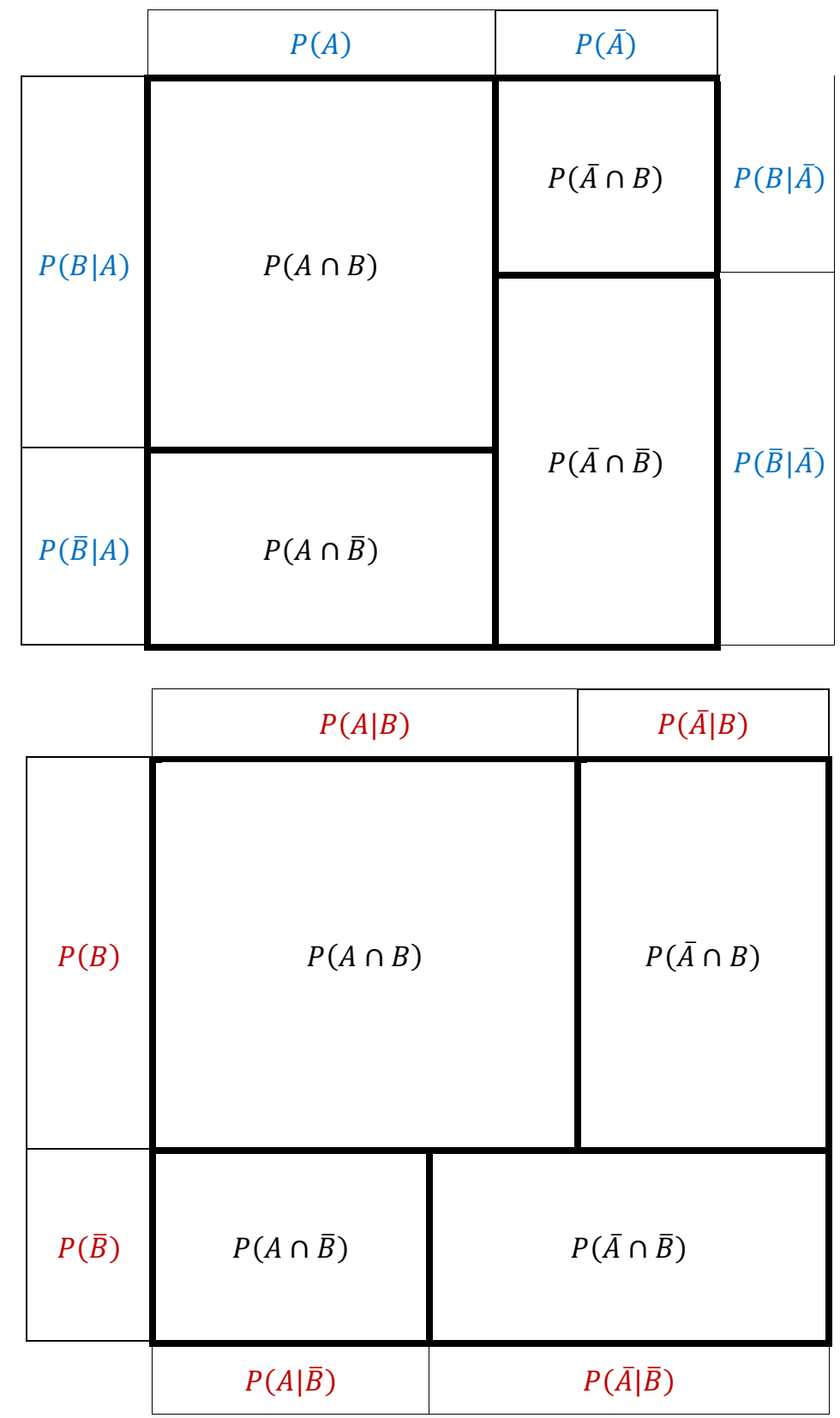

Figure 4. Modification of the Universe of Discourse of Figure 3 so as to be replaced by two different length/area-proportional Karnaugh-Map-like Diagrams. Each diagram is a liaison among the four conjunctive probabilities, two specific marginal probabilities and four specific conditional probabilities. Each map supplies four independent equations, each of which expresses a conjunctive probability (as a product of a conditional probability and a marginal one), as well as two additive relations for conditional probabilities. These twelve basic equations are supplemented by a thirteenth independent equation; which is one of the three equations $(6-8)$ 
International Journal of Mathematical, Engineering and Management Sciences

Vol. 5, No. 5, 787-811, 2020

https://doi.org/10.33889/IJMEMS.2020.5.5.062
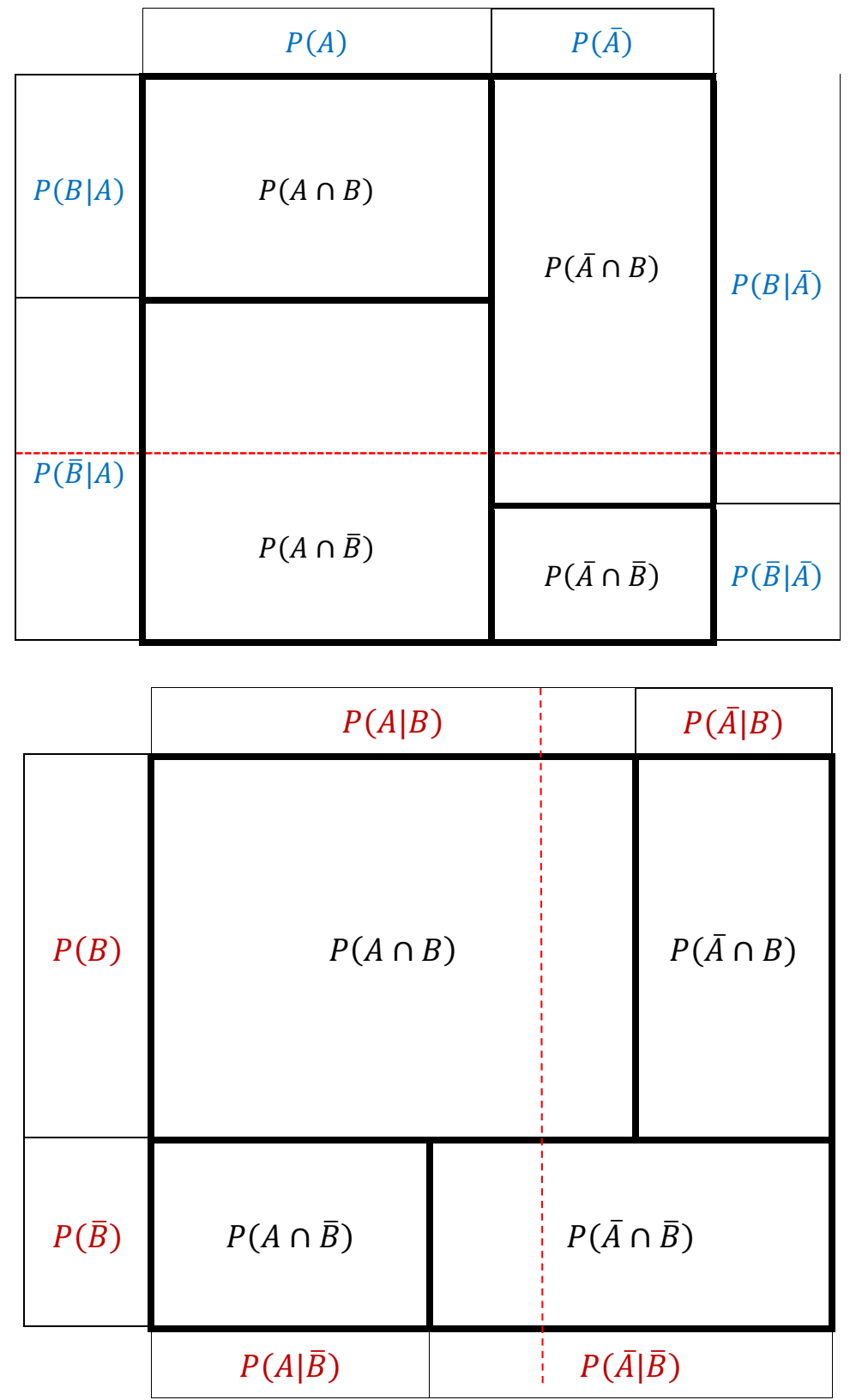

Figure 5. The probability diagrams of Figure 4 redrawn for the case of two mutually disfavoring (weakening) events. $(P(A \mid B)<P(A)),(P(B \mid A)<P(B))$ 
International Journal of Mathematical, Engineering and Management Sciences

Vol. 5, No. 5, 787-811, 2020

https://doi.org/10.33889/IJMEMS.2020.5.5.062

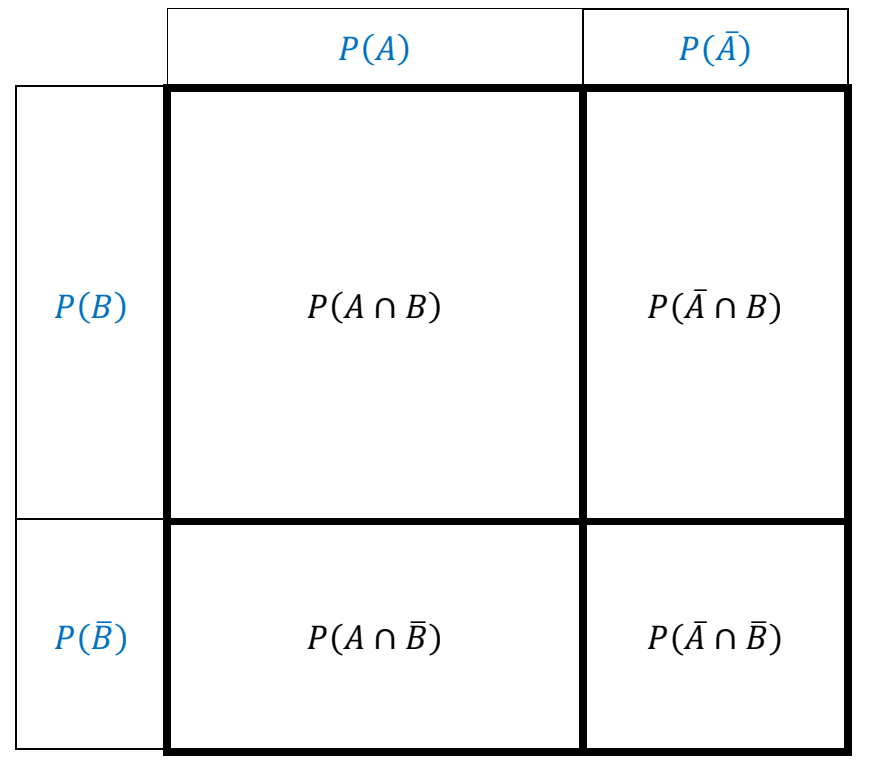

Figure 6. The probability maps of Figure 4 combine into a single map for two independent events $(P(A \mid B)=P(A)),(P(B \mid A)=P(B))$

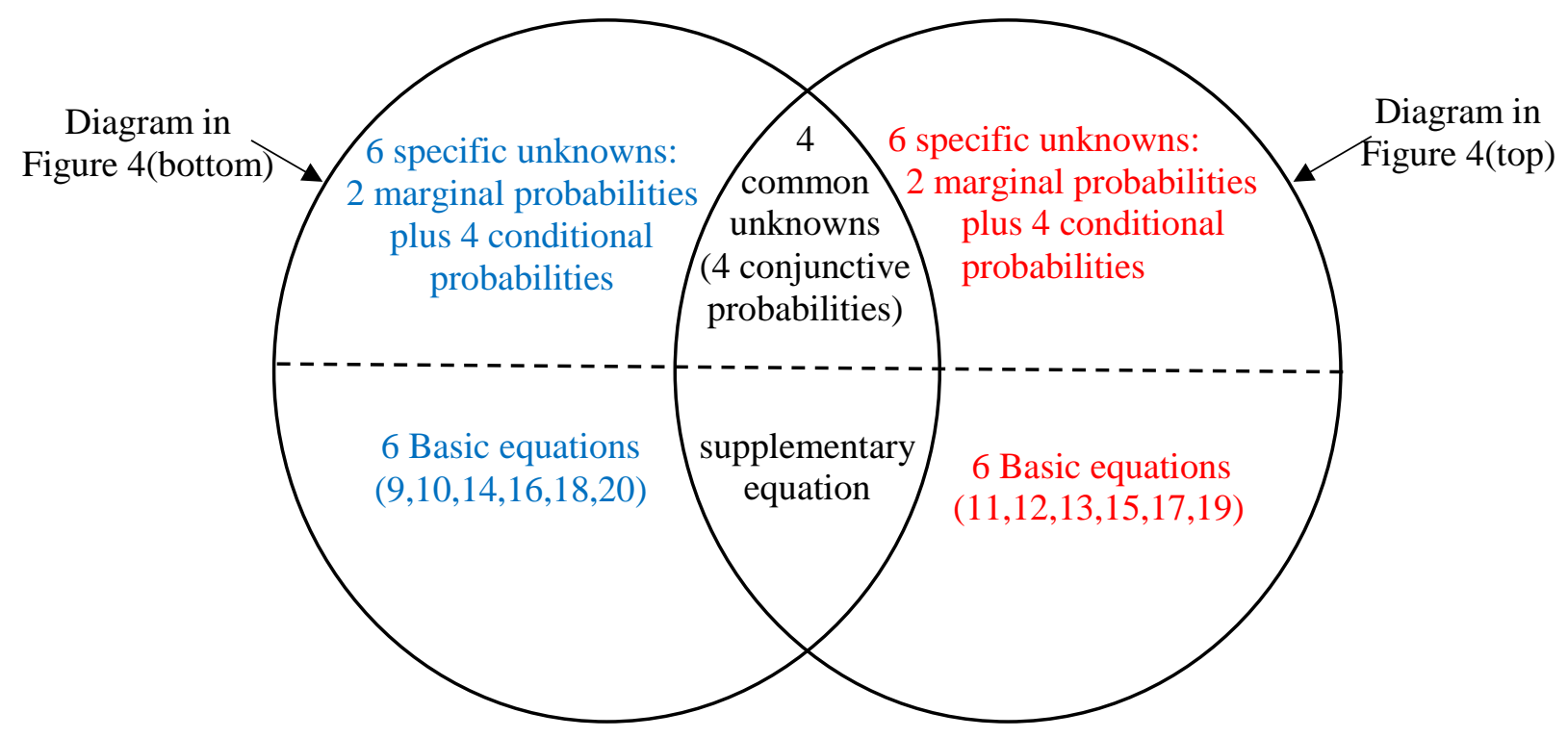

Figure 7. The splitting of unknowns and equations between the two diagrams in Figure 4

Diagrams similar to those in Figures 4 and 5 appeared earlier in many published works including Bertin (1967), Edwards (1972), Hartigan and Kleiner (1981) (under the name of "mosaic"), Oldford and Cherry (2006), Pfannkuch and Budgett (2017) (under the name of "eikosogram," Greek for probability picture), Politzer (2014), Böcherer-Linder et al. (2018), and Böcherer- 
International Journal of Mathematical, Engineering and Management Sciences

Vol. 5, No. 5, 787-811, 2020

https://doi.org/10.33889/IJMEMS.2020.5.5.062

Linder and Eichler (2019). However, we believe that this is the first time a pair of such diagrams is employed as a solution aid for ternary problems of conditional probability.

\section{Ternary Problems of Conditional Probability}

A probability problem is a conditional probability problem if in its formulation at least one of the quantities explicitly mentioned (either as a known quantity or as the unknown to be found) could be interpreted as a conditional probability. A ternary problem of conditional probability is a conditional-probability problem formulated with exactly three known quantities and a single unknown quantity to be solved for. The analysis in this section shows that exactly three independent quantities (no more and no less) should be given in a conditional-probability problem, for this problem to be well formulated and potentially solvable.

A ternary problem of conditional probability that is formulated in the context of a normalized contingency table involves sixteen probabilities, which might be classified as:

- Four marginal probabilities: The two probabilities of the basic events $P(B)$ and $P(A)$ and the two probabilities of the complementary events $P(\bar{B})$ and $P(\bar{A})$.

- Four joint, conjunctive or intersection probabilities: $P(A \cap B), P(\bar{A} \cap B), P(A \cap \bar{B})$ and $P(\bar{A} \cap \bar{B})$.

- Eight conditional probabilities, which are the eight indicators in Figure 3.

There are 19 (redundant) simple or primitive relations among these 16 probabilities, which are exhibited in Table 1. These 19 relations involve the aforementioned 16 probabilities and are represented by what is called a trinomial graph by Huerta $(2009,2014)$ and Huerta et al. (2011), or equivalently by the composite signal flow graph (SFG) in Rushdi et al. (2018) and Rushdi and Talmees $(2018,2019)$. We stress that Table 1 does not include composite or multi-operation probability relations, such as the law of total probability or Bayes' rule. Any of these composite relations is equivalent to a combination of two or more primitive relations. In fact, four versions of the law of total probability emerge through the substitution of (13) and (15) into (2), the substitution of (17) and (19) into (3), the substitution of (14) and (18) into (4), or the substitution of (16) and (20) into (5). Likewise, four versions of Bayes' rule result by equating the R.H.S. of (13) with that of (14), equating the R.H.S. of (15) with that of (16), equating the R.H.S. of (17) with that of (18), and equating the R.H.S. of (19) with that of (20).

It can be seen that the bottom 12 equations in Table 1 (equations (9)-(20), highlighted and stressed in dark blue or dark red) are independent, in the sense that none of them is derivable from the others. We call them basic equations of the ternary problem of conditional probability. The top 4 equations in Table 1 (equations (2)-(5)) are implicit in these 12 basic equations and do not add any independent information, and hence are de-emphasized with a pale light color. By contrast, the next 3 equations (equations (6-8) highlighted in bold green) are independent of the 12 basic equations and hence none of them can be deduced from these basic equations. However, any of the three equations (6-8) might be used to deduce each of the other two (given the 12 basic equations), and any single one of them might supplement the basic equations to form a set of 13 independent equations. Such a set is necessary and sufficient to solve a well-formed ternary problem of conditional probability, in which three of the unknown 16 probabilities are given. 
International Journal of Mathematical, Engineering and Management Sciences

Vol. 5, No. 5, 787-811, 2020

https://doi.org/10.33889/IJMEMS.2020.5.5.062

Table 1. The 19 redundant relations of ternary problems of conditional probability in the contingency table context (classified according to relation type)

\begin{tabular}{|c|c|c|c|}
\hline \multicolumn{2}{|r|}{ Relation type } & \multicolumn{2}{|l|}{ Relation } \\
\hline \multirow{4}{*}{$\begin{array}{l}\text { Additive Relations } \\
\text { (eleven equations) }\end{array}$} & $\begin{array}{l}\text { Two joint probabilities add up to a } \\
\text { marginal probability (four equations) }\end{array}$ & $\begin{array}{l}P(A \cap B)+P(\bar{A} \cap B)=P(B) \\
P(A \cap \bar{B})+P(\bar{A} \cap \bar{B})=P(\bar{B}) \\
P(A \cap B)+P(A \cap \bar{B})=P(A) \\
P(\bar{A} \cap B)+P(\bar{A} \cap \bar{B})=P(\bar{A})\end{array}$ & $\begin{array}{l}(2) \\
(3) \\
(4) \\
(5)\end{array}$ \\
\hline & $\begin{array}{l}\text { Two complementary marginal } \\
\text { probabilities add up to } 1 \text { (two } \\
\text { equations) }\end{array}$ & $\begin{array}{l}P(B)+P(\bar{B})=1 \\
P(A)+P(\bar{A})=1\end{array}$ & $\begin{array}{l}(6) \\
(7)\end{array}$ \\
\hline & $\begin{array}{l}\text { The four joint probabilities add up to } 1 \\
\text { (one equation) }\end{array}$ & $P(A \cap B)+P(\bar{A} \cap B)+P(A \cap \bar{B})+P(\bar{A} \cap \bar{B})=1$ & $(8)$ \\
\hline & $\begin{array}{l}\text { Two complementary conditional } \\
\text { probabilities add up to } 1 \text { (four } \\
\text { equations) }\end{array}$ & $\begin{array}{c}P(A \mid B)+P(\bar{A} \mid B)=1 \\
P(A \mid \bar{B})+P(\bar{A} \mid \bar{B})=1 \\
P(B \mid A)+P(\bar{B} \mid A)=1 \\
P(B \mid \bar{A})+P(\bar{B} \mid \bar{A})=1 \\
\end{array}$ & $\begin{array}{r}(9) \\
(10) \\
(11) \\
(12) \\
\end{array}$ \\
\hline \multirow{4}{*}{\multicolumn{2}{|c|}{$\begin{array}{l}\text { A joint probability is a product of a conditional probability and a } \\
\text { marginal probability (in two different ways) (eight equations) }\end{array}$}} & $\begin{array}{l}P(A \cap B)=P(B \mid A) P(A) \\
P(A \cap B)=P(A \mid B) P(B)\end{array}$ & $\begin{array}{l}(13) \\
(14)\end{array}$ \\
\hline & & $\begin{aligned} P(\bar{A} \cap B) & =P(B \mid \bar{A}) P(\bar{A}) \\
P(\bar{A} \cap B) & =P(\bar{A} \mid B) P(B)\end{aligned}$ & $\begin{array}{l}(15) \\
(16)\end{array}$ \\
\hline & & $\begin{array}{l}P(A \cap \bar{B})=P(\bar{B} \mid A) P(A) \\
P(A \cap \bar{B})=P(A \mid \bar{B}) P(\bar{B})\end{array}$ & $\begin{array}{l}(17) \\
(18)\end{array}$ \\
\hline & & $\begin{array}{c}P(\bar{A} \cap \bar{B})=P(\bar{B} \mid \bar{A}) P(\bar{A}) \\
P(\bar{A} \cap \bar{B})=P(\bar{A} \mid \bar{B}) P(\bar{B})\end{array}$ & $\begin{array}{l}(19) \\
(20)\end{array}$ \\
\hline
\end{tabular}

In passing, we stress that each of the 19 equations of Table 1 has an immediate geometric visualization in one of the two diagrams of Figure 4. Equations (2-5) can be interpreted to mean that the total area of two rectangles with a shared side numerically equals the length of this common side. Equations $(7,11,12)$ state that the overall shape in the top Figure 4 is a square having sides of unit lengths, while equations $(6,9,10)$ assert that the overall shape in the bottom Figure 4 is also a square having sides of unit lengths. Equation (8) indicates that the four rectangles in each of the top and bottom parts of Figure 4 add to a unit square with a total area of unity. Finally, equations (13-20) reveal that the area of any of the four rectangles in each of the top and bottom parts of Figure 4 equals its width times its length.

The visualization capability of the pair of diagrams in Figure 4 is not confined to the primitive probability relations in Table 1 , but it covers composite probability relations as well. According to Figure 4, the marginal probability $P(A)$ is the sum of the two conjunctive probabilities $P(A \cap B)$ and $P(A \cap \bar{B})$, which, equal the products $P(A \mid B) P(B)$ and $P(A \mid \bar{B}) P(\bar{B})$, respectively, according to the bottom part of Figure 4 . The combination of these two observations asserts that

$P(A)=P(A \mid B) P(B)+P(A \mid \bar{B}) P(\bar{B})$,

which is simply a statement of the total probability theorem (TPT) for $P(A)$. The other three cases of the TPT (which express $P(\bar{A}), P(B)$, and $P(\bar{B})$, respectively) are also derivable when the two diagrams of Figure 4 are considered together. The two diagrams also 'co-operate' to derive each of the four various cases of Bayes' rule (BR). For example, the conjunctive probability $P(A \cap B)$ is expressed as $P(B \mid A) P(A)$ according to one diagram, and as $P(A \mid B) P(B)$ according to the other, which amounts to the BR statement: 
International Journal of Mathematical, Engineering and Management Sciences

Vol. 5, No. 5, 787-811, 2020

https://doi.org/10.33889/IJMEMS.2020.5.5.062

$P(B \mid A) P(A)=P(A \mid B) P(B)$.

Cooperation of the two diagrams can be utilized not only for the aforementioned derivations, but also for solving any well-formulated probability problems, including, in particular, wellformulated ternary problems of conditional probabilities (as will be seen in Sec. 6).

A word of caution is warranted about the utility of the pair of diagrams in Figure 4 (or the pair in Figure 5). The immediate applicability of this pair of diagrams might be limited to non-extreme cases only. In fact, the two diagrams assume that each of the eight conditional probabilities in Figure 3 exists, or equivalently, that none of the four marginal probabilities is zero. If this assumption is violated, i.e., if a special limiting case emerges, then appropriate changes are needed while handling the diagrams. For example, consider the case when $P(B)=0$ (while none of $P(A)$ or $P(\bar{A})$ is zero), which depicts the situation of zero apparent prevalence (while the true prevalence is neither zero nor unity). In this case $P(B \mid A)=P(B \mid \bar{A})=0$, and $P(\bar{B})=1$, while neither $P(A \mid B)$ nor $P(\bar{A} \mid B)$ exists. Also $P(A \cap B)=P(\bar{A} \cap B)=0, P(A \mid \bar{B})=P(A \cap \bar{B})=$ $P(A)$ while $P(\bar{A} \mid \bar{B})=P(\bar{A} \cap \bar{B})=P(\bar{A})$. This means that event $B$ totally disappears from each of the diagrams in Figure 4 (or from each in Figure 5 ). In fact, both diagrams degenerate into a unit square split into two rectangles representing $P(A)$ and $P(\bar{A})$, respectively. This is a case where no ternary problem of conditional probability could be formulated.

\section{Solution of Ternary Problems of Conditional Probability}

A complete solution of a ternary problem of conditional probability necessitates finding 16 probabilities (given exactly 3 of them, which are independent) via 12 basic equations together with one out of three supplementary equations. Figure 7 shows that the combined use of the diagrams in Figure 4 constitutes a divide-and-conquer methodology for problem solution, since we can split the 16 probabilities and the 13 independent equations almost evenly (with a minimal overlapping) between the two diagrams in Figure 4. In fact, each of the two diagrams deals with 10 probabilities as follows. Each diagram takes care individually and separately of a disjoint set of 6 probabilities, with the two sets together constituting 12 of the 16 probabilities under consideration. The remaining 4 probabilities are handled in a shared-fashion by both diagrams. On the other hand, each of the two diagrams provides individually and separately 6 of the 12 basic equations. The top diagram in Figure 4 addresses equations $(11-13,15,17,19)$ distinguished in bold red, while the bottom diagram of Figure 4 visualizes equations $(9,10,14,16,18,20)$ emphasized in bold blue. The 13th independent equation is supplied as a supplementary equation by either diagram (actually either diagram can provide two 'dependent' equations of the three potential supplementary equations (6-8) shown in dark green). This means that each diagram has 7 equations to process 10 unknowns.

Now, we have two possibilities:

1- The given 3 unknowns are totally among the 10 unknowns of one particular diagram:

In this case, the diagram supplied with the 3 unknown values ends up with 7 equations in 7 unknowns. This diagram is completely solvable as is, and when solved it imparts values of the 4 conjunctive probabilities to the other diagram making it also completely solvable. This case corresponds to the case of "arithmetic reading" in Huerta $(2009 ; 2014)$ and Rushdi and Talmees $(2018 ; 2019)$, while the next case corresponds to what is called algebraic reading.

2- The 3 given unknowns are split such that 2 are among the 10 unknowns of one diagram, with the remaining third unknown being among the 10 unknowns of the other diagram: 
International Journal of Mathematical, Engineering and Management Sciences

Vol. 5, No. 5, 787-811, 2020

https://doi.org/10.33889/IJMEMS.2020.5.5.062

This case does not allow direct arithmetic solution but requires algebraic formulation. The diagram with exactly two given unknowns is not solvable because it has 7 equations against 8 unknowns. However, if we assign an arbitrary value " $X$ " to an appropriate variable among these 8 unknowns, we can assume that we are left with 7 unknowns only and can solve the diagram completely in terms of $X$. The other diagram can also be solved completely in terms of $X$ with (at least) one of the unknowns being computed twice. Hence, an equation in $X$ emerges and might be solved, so that all values computed in terms of $X$ are now given final numerical values.

An offshoot benefit of using the diagrams in Figure 4 is that they clarify why arithmetic processing might suffice to handle a ternary problem of conditional probability, and also identify conditions for algebraic reading to become a must. In this latter case, the set of candidate unknowns such that one of which is to be assigned an arbitrary symbolic value " $X$ " is considerably narrowed.

\section{Example 1:}

Assume we are given the three probabilities $P(A)=0.2, P(B \mid A)=0.9$, and $P(B \mid \bar{A})=0.1$. These three values belong solely to the top diagram in Figure 4 , and hence a purely arithmetic solution is possible, as shown in Figure 8, in which various steps are assigned Roman numerals to facilitate the reading of the solution.

\section{Example 2:}

Now, we are given the three conditional probabilities $P(B \mid A)=0.8, P(A \mid B)=0.4$, and $P(A \mid \bar{B})=$ 0.1 . The first of these three values belong to the top diagram in Figure 4, while the remaining two belong to the bottom diagram in Figure 4 . We supplement this latter diagram by an arbitrarily assumed value $X$ for a suitable one of its remaining unknowns, namely $P(B)$. We perform the computations in Figure 9 in order to express $P(A \cap B)$ (on the top diagram) twice in terms of $X$. Hence, we obtain the equation:

$0.4 X=0.08+0.24 X$,

which yields $X=0.5$. A second traversal of all the probabilities in Figure 9 to substitute the value of $X$ produces the desired final numerical values. 
International Journal of Mathematical, Engineering and Management Sciences

Vol. 5, No. 5, 787-811, 2020

https://doi.org/10.33889/IJMEMS.2020.5.5.062
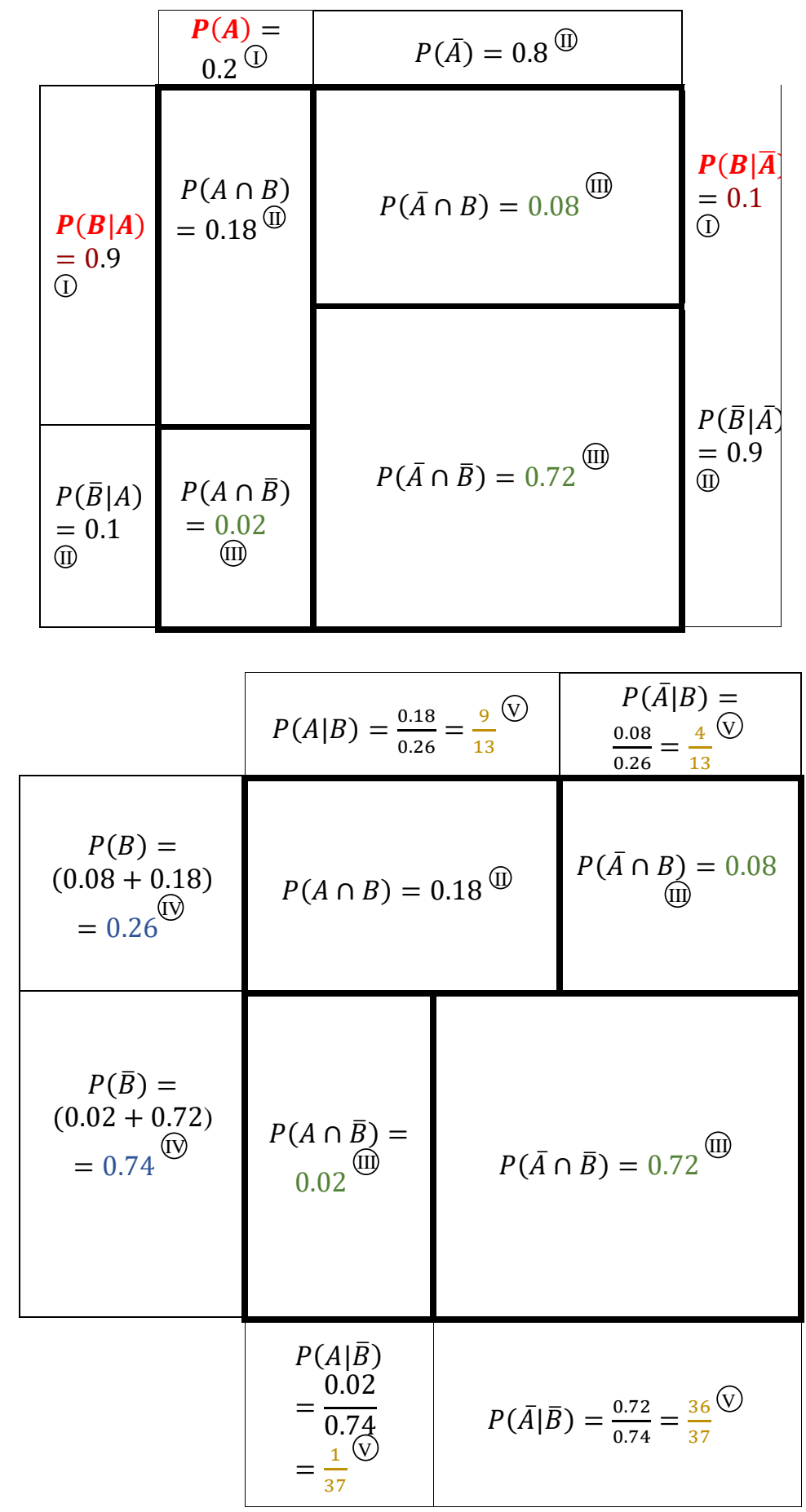

Figure 8. Arithmetic solution of a ternary problem of conditional probability given the three known values

$$
P(A)=0.2, P(B \mid A)=0.9, \text { and } P(B \mid \bar{A})=0.1
$$


International Journal of Mathematical, Engineering and Management Sciences

Vol. 5, No. 5, 787-811, 2020

https://doi.org/10.33889/IJMEMS.2020.5.5.062

\begin{tabular}{|c|c|c|c|}
\hline & $\begin{array}{l}P(A)= \\
0.1+0.3 X \\
=0.25\end{array}$ & $\begin{aligned} P(\bar{A}) & =0.9-0.3 X^{\text {IV }} \\
& =0.75\end{aligned}$ & \\
\hline $\begin{aligned} & P(B \mid A) \\
= & 0.8^{\mathrm{I}}\end{aligned}$ & $\begin{array}{c}P(A \cap B) \\
=0.4 X \\
= \\
0.08+\end{array}$ & $\begin{array}{c}P(\bar{A} \cap B)=0.6 X^{\text {III }} \\
=0.3\end{array}$ & $\begin{array}{l}P(B \mid \bar{A})= \\
\frac{0.6 X}{0.9-0.3 X} \\
=0.4\end{array}$ \\
\hline & & \multirow[b]{2}{*}{$\begin{aligned} P(\bar{A} \cap \bar{B}) & =0.9(1-X)^{\text {IIII) }} \\
= & 0.45\end{aligned}$} & \multirow{2}{*}{$\begin{array}{l}P(\bar{B} \mid \bar{A})= \\
\frac{0.9-0.9 X}{0.9-0.3 X} \\
=0.6\end{array}$} \\
\hline $\begin{aligned} & P(\bar{B} \mid A) \\
= & 0.2^{\text {III }}\end{aligned}$ & $\begin{array}{c}P(A \cap \bar{B}) \\
= \\
0.1(1-X) \\
\text { (III) } \\
=0.05\end{array}$ & & \\
\hline
\end{tabular}

\begin{tabular}{|c|c|c|c|}
\hline & \multicolumn{2}{|c|}{$P(A \mid B)=0.4$} & $\begin{array}{c}P(\bar{A} \mid B) \\
\text { (III) }\end{array}=0.6$ \\
\hline 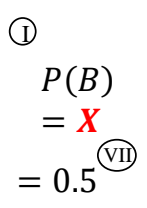 & \multicolumn{2}{|c|}{$\begin{array}{c}P(A \cap B)=0.4 X^{\text {III }} \\
=0.2\end{array}$} & $\begin{array}{c}P(\bar{A} \cap B)= \\
0.6 X^{\text {III }} \\
\quad=0.3\end{array}$ \\
\hline \multirow[t]{2}{*}{$\begin{array}{c}\text { III } \\
P(\bar{B})= \\
(1-X) \\
= \\
0.5^{\text {VIII }}\end{array}$} & $\begin{aligned} & P(A \cap \bar{B}) \\
= & 0.1(1-X) \\
= & 0.05\end{aligned}$ & \multicolumn{2}{|c|}{$\begin{aligned} P(\bar{A} \cap \bar{B}) & =0.9(1-X)^{\text {IIII }} \\
= & 0.45\end{aligned}$} \\
\hline & $\begin{array}{c}\text { (1) } P(A \mid \bar{B})= \\
0.1\end{array}$ & \multicolumn{2}{|c|}{$P(\bar{A} \mid \bar{B})=0.9^{\text {III }}$} \\
\hline
\end{tabular}

Figure 9. Algebraic solution of a ternary problem of conditional probability given the three known values $P(B \mid A)=0.8, P(A \mid B)=0.4$, and $P(A \mid \bar{B})=0.1$. Since these values are split between the two diagrams, we assume a value of $X$ for $P(B)$ and behave as if we have three knowns in the lower map 
International Journal of Mathematical, Engineering and Management Sciences

Vol. 5, No. 5, 787-811, 2020

https://doi.org/10.33889/IJMEMS.2020.5.5.062

\section{Example 3:}

This example derives formulas for $P P V_{i j}(P(A \mid B))$ and $N P V_{i j}(P(\bar{A} \mid \bar{B}))$ in terms of $(a)$ the true prevalence $($ Prev $=P(A)=$ Probability of disease presence according to the gold standard $)$, (b) the sensitivity $\operatorname{Sens}_{i j}(P(B \mid A))$, and (c) the specificity $\operatorname{Spec}_{i j}(P(\bar{B} \mid \bar{A}))$. The three given quantities all belong to the top diagram in Figure 4. Therefore, a symbolic arithmetic solution is possible, the initial steps of which are shown in Figure 10. The computations in Figure 10 might be continued to obtain the apparent prevalence $\operatorname{Prev}^{\prime}(P(B))$ and its complement $P(\bar{B})$ as

$$
\begin{aligned}
& P(B)=\text { Prev }^{\prime}=\text { Sens }_{i j} \text { Prev }+\left(1-\text { Spec }_{i j}\right)(1-\text { Prev }) . \\
& P(\bar{B})=\left(1-\text { Sens }_{i j}\right) \text { Prev }+ \text { Spec }_{i j}(1-\text { Prev }) .
\end{aligned}
$$

Subsequently, the $P P V_{i j}$ and $N P V_{i j}$ are obtained as

$$
\begin{aligned}
& P P V_{i j}=P(A \mid B)=\frac{\operatorname{Sens}_{i j} \text { Prev }}{\text { Sens }_{i j} \text { Prev }_{\left(1-\text { Spec }_{i j}\right)(1-\text { Prev })}} \\
& N P V_{i j}=P(\bar{A} \mid \bar{B})=\frac{\operatorname{Spec}_{i j}(1-\text { Prev })}{\left(1-\text { Sens }_{i j}\right) \text { Prev }_{\text {Spec }}(1-\text { Prev })}
\end{aligned}
$$

The expressions (24)-(27) are well known TPT and BR results (see, e.g. Kelly et al., 2008; Rushdi and Rushdi, 2018). Likewise, we might use algebraic techniques to express the sensitivity and specificity in terms of prevalence and predictive values as follows (in agreement with Kelly et al. (2008))

$$
\begin{aligned}
& \text { Sens }_{i j}=P(B \mid A)=\frac{P P V_{i j} \text { Prev }^{\prime}}{\text { Prev }}=\frac{P P V_{i j}\left(N P V_{i j}+\text { Prev }-1\right)}{\left(N P V_{i j}+P P V_{i j}-1\right) \text { Prev }} \\
& \text { Spec }_{i j}=P(\bar{B} \mid \bar{A})=\frac{N P V_{i j}\left(1-\text { Prev }^{\prime}\right)}{(1-\text { Prev })}=\frac{N P V_{i j}\left(P P V_{i j}-\text { Prev }\right)}{\left(N P V_{i j}+P P V_{i j}-1\right)(1-\text { Prev })}
\end{aligned}
$$

We might exploit (24) and (28) to express the true and apparent prevalence via the 'dual' relations

$$
\begin{gathered}
\text { Prev }=\frac{\left(\text { Spec }_{i j}+\text { Prev }^{\prime}-1\right)}{\left(\text { Spec }_{i j}+\text { Sens }_{i j}-1\right)} \\
\text { Prev }^{\prime}=\frac{\left(N P V_{i j}+\text { Prev }^{-1}\right)}{\left(N P V_{i j}+P P V_{i j}-1\right)}
\end{gathered}
$$


International Journal of Mathematical, Engineering and Management Sciences

Vol. 5, No. 5, 787-811, 2020

https://doi.org/10.33889/IJMEMS.2020.5.5.062

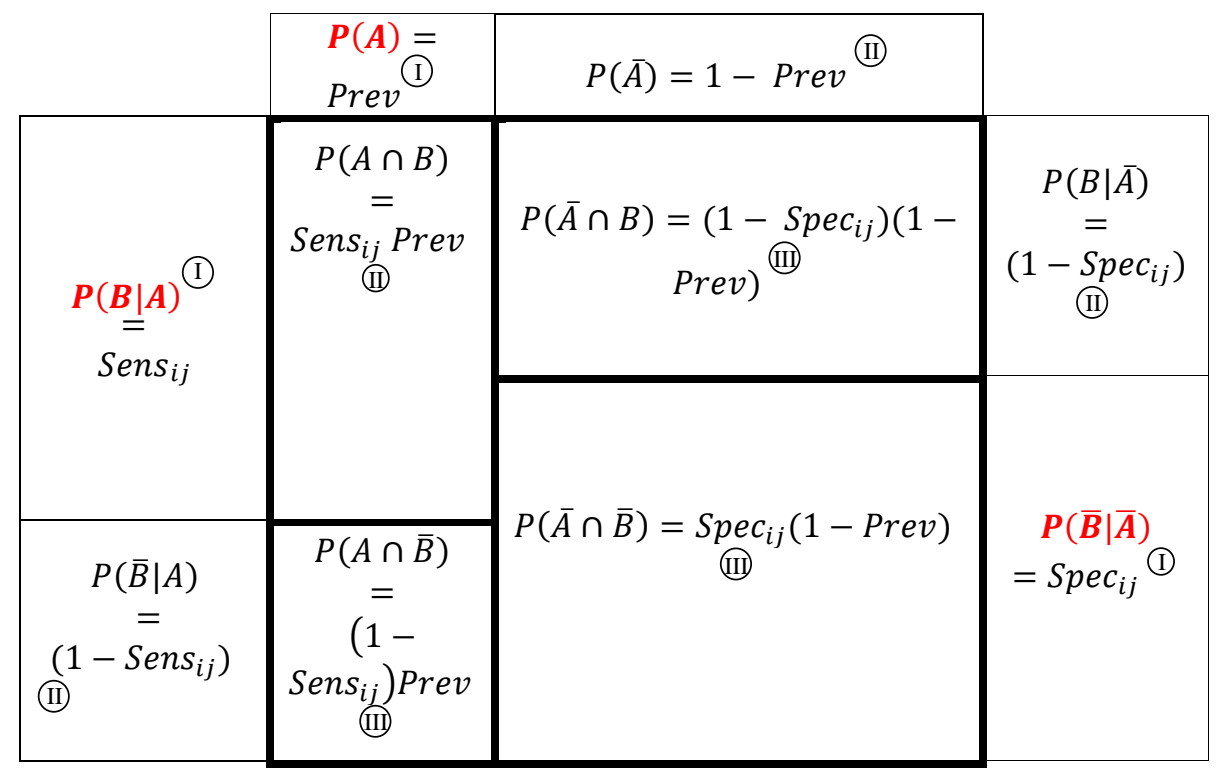

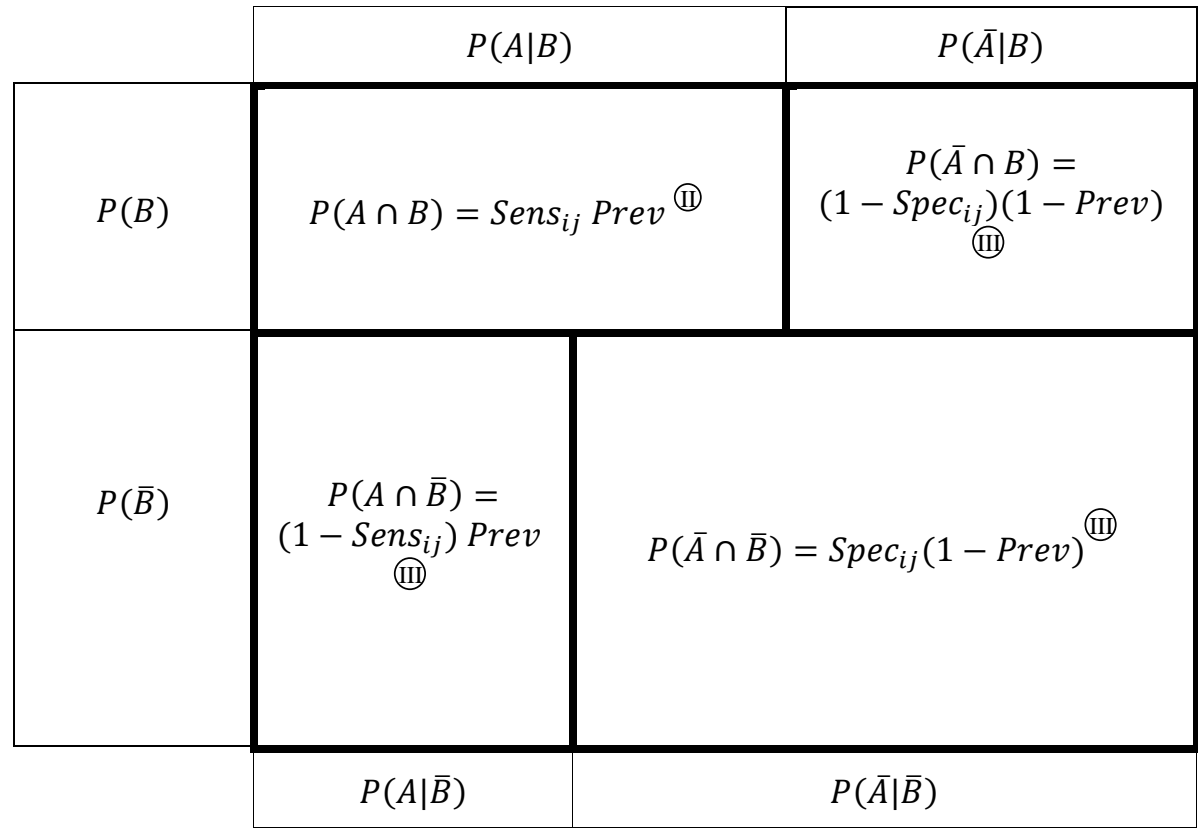

Figure 10. Derivation of expressions for the predictive values $P P V_{i j}(P(A \mid B))$ and $N P V_{i j}(P(\bar{A} \mid \bar{B}))$ in terms of the prevalence $(P(A)), \operatorname{Sens}_{i j}(P(B \mid A))$ and $\operatorname{Spec}_{i j}(P(\bar{B} \mid \bar{A}))$

\section{Example 4:}

This example (demonstrated by Figure 11) shows how we might use algebra to compute the sensitivity in terms of the specificity and predictive values (in the absence of knowledge about the prevalence). Here, we are given the three conditional probabilities $P(\bar{B} \mid \bar{A})=\operatorname{Spec}_{i j}, P(A \mid B)=$ $P P V_{i j}$, and $P(\bar{A} \mid \bar{B})=N P V_{i j}$. The first of these three values belong to the top diagram in Figure 4, while the remaining two belong to the bottom diagram in Figure 4. We supplement this latter 
International Journal of Mathematical, Engineering and Management Sciences

Vol. 5, No. 5, 787-811, 2020

https://doi.org/10.33889/IJMEMS.2020.5.5.062

diagram by an arbitrarily assumed value $X$ for the apparent prevalence $(P(B))$. Calculations in Figure 11 are followed by the following computations

$$
\begin{aligned}
& P(\bar{A})=\frac{X\left(1-P P V_{i j}\right)}{1-\operatorname{Spec}_{i j}}=\frac{(1-X) N P V_{i j}}{\operatorname{Spec}_{i j}} \\
& \frac{X}{1-X}=\frac{1-\operatorname{Spec}_{i j}}{\operatorname{Spec}_{i j}} \frac{N P V_{i j}}{1-P P V_{i j}} \\
& X=\frac{\left(1-\operatorname{Spec}_{i j}\right) N P V_{i j}}{\left(1-\operatorname{Spec}_{i j}\right) N P V_{i j}+\operatorname{Spec}_{i j}\left(1-P P V_{i j}\right)} \\
& P(\bar{A})=\frac{N P V_{i j}\left(1-P P V_{i j}\right)}{\left(1-\operatorname{Spec}_{i j}\right) N P V_{i j}+\operatorname{Spec}_{i j}\left(1-P P V_{i j}\right)} \\
& P(A)=\frac{\left[1-\left(N P V_{i j}+P P V_{i j}\right)\right] \operatorname{Spec}_{i j}+N P V_{i j} P P V_{i j}}{\left(1-\operatorname{Spec}_{i j}\right) N P V_{i j}+\operatorname{Spec}_{i j}\left(1-P P V_{i j}\right)} \\
& \operatorname{Sens}_{i j}=\frac{X P P V_{i j}}{P(A)}=\frac{P P V_{i j} N P V_{i j}\left[1-\operatorname{Spec}_{i j}\right]}{P P V_{i j} N P V_{i j}+\operatorname{Spec}_{i j}\left[1-P P V_{i j}-N P V_{i j}\right]}
\end{aligned}
$$

This equation might be rewritten as

$$
P(B \mid A)=\frac{P(A \mid B) P(\bar{A} \mid \bar{B})[1-P(\bar{B} \mid \bar{A})]}{P(A \mid B) P(\bar{A} \mid \bar{B})+P(\bar{B} \mid \bar{A})[1-P(A \mid B)-P(\bar{A} \mid \bar{B})]}
$$

Now, if we use appropriate symbol interchanges, we obtain

$$
\begin{aligned}
& P(\bar{B} \mid \bar{A})=\frac{P(A \mid B) P(\bar{A} \mid \bar{B})[1-P(B \mid A)]}{P(A \mid B) P(\bar{A} \mid \bar{B})+P(B \mid A)[1-P(A \mid B)-P(\bar{A} \mid \bar{B})]} \\
& P(A \mid B)=\frac{P(B \mid A) P(\bar{B} \mid \bar{A})[1-P(\bar{A} \mid \bar{B})]}{P(B \mid A) P(\bar{B} \mid \bar{A})+P(\bar{A} \mid \bar{B})[1-P(B \mid A)-P(\bar{B} \mid \bar{A})]} \\
& P(\bar{A} \mid \bar{B})=\frac{P(B \mid A) P(\bar{B} \mid \bar{A})[1-P(A \mid B)]}{P(B \mid A) P(\bar{B} \mid \bar{A})+P(A \mid B)[1-P(B \mid A)-P(\bar{B} \mid \bar{A})]}
\end{aligned}
$$

which translate to the following expressions

$$
\begin{aligned}
& \operatorname{Spec}_{i j}=\frac{P P V_{i j} * N P V_{i j}\left[1-\operatorname{Sens}_{i j}\right]}{P P V_{i j} * N P V_{i j}+\operatorname{Sens}_{i j}\left[1-P P V_{i j}-N P V_{i j}\right]} \\
& P P V_{i j}=\frac{\operatorname{Sens}_{i j} * \operatorname{Spec}_{i j}\left[1-N P V_{i j}\right]}{\operatorname{Sens}_{i j} * \operatorname{Sec}_{i j}+N P V_{i j}\left[1-\operatorname{Sens}_{i j}-\operatorname{Spec}_{i j}\right]} \\
& N P V_{i j}=\frac{\operatorname{Sens}_{i j} * \operatorname{Spec}_{i j}\left[1-P P V_{i j}\right]}{\operatorname{Sens}_{i j} * \operatorname{Spec}_{i j}+P P V_{i j}\left[1-\operatorname{Sens}_{i j}-\operatorname{Spec}_{i j}\right]}
\end{aligned}
$$


International Journal of Mathematical, Engineering and Management Sciences

Vol. 5, No. 5, 787-811, 2020

https://doi.org/10.33889/IJMEMS.2020.5.5.062

\begin{tabular}{|c|c|c|c|}
\hline & $P(A)$ & $P(\bar{A})$ & \\
\hline$P(B \mid A)$ & $\begin{aligned} & P(A \cap B) \\
= & X * P P V_{i j} \\
& \text { (ID) }\end{aligned}$ & $P(\bar{A} \cap B)=X\left(1-P P V_{i j}\right)$ & $\begin{array}{c}P(B \mid \bar{A}) \\
= \\
(1- \\
\left.\text { Spec }_{i j}\right) \\
\text { (II) }\end{array}$ \\
\hline$P(\bar{B} \mid A)$ & $\begin{array}{c}P(A \cap \bar{B}) \\
= \\
(1- \\
X)(1-\overline{1} \\
\left.N P V_{i j}\right)\end{array}$ & $P(\bar{A} \cap \bar{B})=\underset{\text { (IV) }}{(1-X) N P V_{i j}}$ & $\begin{aligned} & \boldsymbol{P}(\overline{\boldsymbol{B}} \mid \overline{\boldsymbol{A}}) \\
= & \operatorname{Spec}_{i j} \text { (I) }\end{aligned}$ \\
\hline
\end{tabular}

\begin{tabular}{|c|c|c|c|}
\hline & \multicolumn{2}{|c|}{$P(A \mid B)=P P V_{i j}$} & $\begin{array}{c}P(\bar{A} \mid B)= \\
1-P P V_{i j}\end{array}$ \\
\hline $\begin{aligned} & P(B) \\
= & X\end{aligned}$ & \multicolumn{2}{|c|}{$P(A \cap B)=X * P P V_{i j}$} & $\begin{array}{c}P(\bar{A} \cap B)= \\
X\left(1-P P V_{i j}\right)\end{array}$ \\
\hline \multirow[t]{2}{*}{$\begin{aligned} & P(\bar{B}) \\
= & (1-X)^{\text {III }}\end{aligned}$} & $\begin{array}{c}P(A \cap \bar{B})= \\
(1-X)(1- \\
\left.N P V_{i j}\right)\end{array}$ & \multicolumn{2}{|c|}{$P(\bar{A} \cap \bar{B})=(1-X) N P V_{i j}{ }^{\text {III }}$} \\
\hline & $\begin{array}{c}P(A \mid \bar{B})= \\
\left(1-N P V_{i j}\right)\end{array}$ & & $=N P V_{i j}$ \\
\hline
\end{tabular}

Figure 11. Steps for algebraic computing of the sensitivity $\left(\operatorname{Sens}_{i j}=P(B \mid A)\right)$ in terms of the specificity $\left(\operatorname{Spec}_{i j}=P(\bar{B} \mid \bar{A})\right)$, the positive predictive value $\left(P P V_{i j}=P(A \mid B)\right)$ and negative predictive value $\left(N P V_{i j}=\right.$ $P(\bar{A} \mid \bar{B})$ ) under the assumption that no division by zero is committed

Equations $(36,38-40)$ express each of the four most prominent indicators of diagnostic testing (Sensitivity, Specificity, Positive Predictive Value, and Negative Predictive Value) solely in terms of the other three. They seem to be novel ones. We have not come across them anywhere in the open literature. Of course, these equations are valid (in their present forms) only in regular or non-limiting cases and provided no division by zero is involved. 
International Journal of Mathematical, Engineering and Management Sciences

Vol. 5, No. 5, 787-811, 2020

https://doi.org/10.33889/IJMEMS.2020.5.5.062

\section{Application to the Assessment of Prevalence of the COVID-19 Pandemic}

The ongoing epidemic of the novel coronavirus (now called SARS-CoV-2, causing the disease COVID-19) seems to have expanded from the Wuhan province in China, but has definitely reached almost any inhabited area on earth (Goldstein and Burstyn, 2020; Guo et al., 2020; Lipsitch et al., 2020; Li et al., 2020; Park et al., 2020; Rothan and Byrareddy, 2020; Yang et al., 2020; Wu et al., 2020). This fatal disease, being a catastrophic threat of great global public-health concern, is expected to have diverse grave (and potentially irreversible) consequences. Therefore, research concerning various aspects of epidemiology, in general, with a stress on epidemiological facets, features and aspects related to Covid-19, in particular, might pay off handsomely in improving the response of humanity to this epidemic, now characterized as a pandemic by the World Health Organization (2020). This paper constitutes a modest effort that falls under the umbrella of such research. The paper offers good insight as well as efficient computational techniques for the ternary problem of conditional probability as formulated in the context of a contingency table, a well-known and prominent epidemiology problem. The insight gained and the techniques presented might be instrumental in characterizing diseases and epidemics as well as in assessing (and even in constructing) artificial-intelligence (AI) models for detecting them. We note that though COVID-19 made its first appearance in our world only a few months ago, already several papers on its detection via AI techniques have already appeared (see, e.g. Chowdhury et al., 2020; Li et al., 2020; Salman et al., 2020; Sethy et al., 2020).

We devote the rest of this section to a timely (though narrow) application of the methods developed herein, namely, to the issue of assessment of the true prevalence of the COVID-19 pandemic (and any similar disease) at any instance of time (worldwide or within a specific region), a prelude to determining the rate at which the disease is spreading or retreating. Such an assessment is naturally based on implementing certain tests, which (albeit adequate) are somewhat imperfect and cannot be elevated to the status of a gold standard. The present models indicate that limited (rather than imperfect) testing should be the main source of concern at this stage (Goldstein and Burstyn, 2020; Peto, 2020). Tests (even if imperfect) facilitate the identification (and isolation) of infected individuals, guide the medical treatment that they receive, allow the tracing and quarantining of their contacts, thereby leading to prudent allocation of medical resources, and to quick understanding of this unprecedented phenomenon.

Let us first consider the extreme case of $P(B)=0$, considered earlier in Sec. 5. Here, Prev ${ }^{\prime}=0$, $S_{e n s} s_{i j}=0$, and $\operatorname{Spec}_{i j}=1$, and hence according to (30), the true prevalence Prev is 0/0, which is an undefined quantity. This result manifests the correct (but entirely absurd or even useless) conclusion that if we perform no testing, then we have no clue whatsoever as to what the true prevalence of the pandemic is. Of course, this extreme case does not resemble the actual situation of COVID-19 in any country. But it might serve as a first cut at (or an engineering approximation of) an assessment of the situation in a few countries, which lack the resources or the desire to perform testing on a plausible part of the population (including at least symptomatic individuals as well as those who had been in direct contact with them). We cannot overstress the importance of global and early testing (even when imperfect) in a pandemic such as COVID-19. For a typical test of that pandemic, Goldstein and Burstyn (2020) assume that a plausible value of Sens $_{i j}$ is between 0.6 and 0.9, while $\operatorname{Spec}_{i j}$ might be held high between 0,9 and 1.0. In contrast to perfect testing $\left(\operatorname{Sens}_{i j}=1, \operatorname{Spec}_{i j}=1\right)$ for which $\left(\right.$ Prev $\left.=\operatorname{Prev}^{\prime}\right)$, an available imperfect test produces (according to Equation (30)) the following upper bound on true prevalence, 
International Journal of Mathematical, Engineering and Management Sciences

Vol. 5, No. 5, 787-811, 2020

https://doi.org/10.33889/IJMEMS.2020.5.5.062

$$
\text { Prev } \leq \frac{\text { Prev' }}{0.6}=1.667 \text { Prev }^{\prime}
$$

Equation (44) means that typical tests for COVID-19 might underestimate prevalence of the pandemic to $60 \%$ of its actual value. While our estimate of the pandemic prevalence is somewhat hampered by testing imperfection, it is seriously undermined by the lack of extensive or global testing. To see this in mathematical terms, let us assume that the total population consists of $N_{u}$ and $N_{t}$ untested and tested individuals, respectively. Let $\operatorname{Prev}_{u}$ denote the pandemic true prevalence among those that have not been tested and let $\operatorname{Prev}_{t}$ denote the corresponding value for those who have been tested. According to our previous discussion, $\operatorname{Prev}_{u}$ is totally unknown, apart from the fact that it is a probability, and hence it is such that

$$
0 \leq \operatorname{Prev}_{u} \leq 1
$$

Our previous equation (44) pertains solely to the tested population. To stress this fact, we rewrite it with its variables qualified with the subscript $t$ (for tested)

$\operatorname{Prev}_{t} \leq 1.667$ Prev $_{t}^{\prime}$

Now, we can express the true prevalence Prev among the general mixed population as a weighted sum of the corresponding values $\operatorname{Prev}_{u}$ and $\operatorname{Prev}_{t}$ among the untested and tested populations, respectively

$$
\text { Prev }=\frac{N_{u} \text { Prev }_{u}+N_{t} \text { Prev }_{t}}{N_{u}+N_{t}}
$$

Define the non-negative 'dimensionless' parameter $\rho=N_{t} / N_{u}$ that denotes testing intensity. This parameter attains its minimum value of 0 in the case of no testing at all, and it increases without bound (tends to infinity) when perfect global testing is realized. This parameter might be invoked in (46) as follows

Prev $=\frac{\text { Prev }_{u}+\rho \text { Prev }_{t}}{1+\rho}$

When (46a) is combined with (45), we obtain bounds for the prevalence in the general population as follows

$$
\frac{\rho \operatorname{Prev}_{t}}{1+\rho} \leq \operatorname{Prev} \leq \frac{1+\rho \text { Prev }_{t}}{1+\rho}
$$

Equation (47) sets bounds on the pandemic prevalence among the general population, in terms of two parameters: (a) the true prevalence Prev $v_{t}$ among those tested, and (b) the testing intensity $\rho$. The interval between these bounds is unacceptably wide for small $\rho$, but narrows gradually as $\rho$ increases.

\section{Conclusions}

This paper provided a divide-and-conquer methodology for solving a ternary problem of conditional probability associated with a two-by-two contingency matrix, through the utilization of two length/area-proportional normalized variants of such a matrix. The methodology is 
International Journal of Mathematical, Engineering and Management Sciences

Vol. 5, No. 5, 787-811, 2020

https://doi.org/10.33889/IJMEMS.2020.5.5.062

particularly useful in various areas of diagnostic testing such as clinical or epidemiological testing, though it is still conveniently applicable in other types of problems of general nature involving conditional probabilities. The paper explains why and how a conditional-probability problem (with exactly three appropriate quantities being given or pre-specified) can be solved. The paper also identifies the case when an arithmetic solution is possible and differentiates this case from the case when an algebraic solution is warranted. The methodology proposed herein can be used to recover all known relations involving quantities pertinent to or derivable from the two-by-two contingency table. As a particularly significant offshoot, this methodology shows that the four most prominent indicators of diagnostic testing (Sensitivity, Specificity, Positive Predictive Value, and Negative Predictive Value) constitute three rather than four independent quantities. This observation is virtually unheard of, though it is implicit in earlier solutions of the ternary problem of conditional probability.

The contribution of this paper to the diagnostic testing aspects of mathematical epidemiology culminates in a timely application to and a discussion of the contemporary world-wide COVID19 pandemic. An important observation made herein is that our assessment of the pandemic prevalence is affected by test imperfection, which tends to cause an inadvertent underestimation of the prevalence value. However, this is a mild problem that can be tolerated or even accommodated by using a 'factor of safety.' A more serious problem is that of using a testing intensity that is too low to provide concrete information with some acceptable uncertainty.

\section{Conflict of Interest}

The authors assert that no conflict of interest exists.

\section{Acknowledgement}

This work is funded by the Deanship of Scientific Research (DSR), King Abdulaziz University, Jeddah. Therefore, the authors acknowledge, with thanks, the DSR for their financial and technical support. The first-named author (AMAR) is gratefully indebted to Dr. Rufaidah Rushdi, of Kasr Al-Ainy Faculty of Medicine (Cairo University, Arab Republic of Egypt) for stimulating discussions concerning epidemiological testing and its various measures, and for proposing an application of our results to COVID-19.

\section{References}

Amin, M.N., Rushdi, M.A., Marzaban, R.N., Yosry, A., Kim, K., \& Mahmoud, A.M. (2019). Waveletbased computationally-efficient computer-aided characterization of liver steatosis using conventional B-mode ultrasound images. Biomedical Signal Processing and Control, 52, 84-96.

Anderson, T.W., \& Finn, J.D. (1996). Summarizing multivariate data: association between categorical variables. In The New Statistical Analysis of Data (pp. 177-230). Springer, New York.

Bertin, J. (1967). Sémiologie graphique. paris, gauthier-villars. Translation. as Semiology of Graphics (1983). Wisconsin: University of Wisconsin Press.

Böcherer-Linder, K., \& Eichler, A. (2019). How to improve performance in Bayesian inference tasks: a comparison of five visualizations. Frontiers in Psychology, 10, Article 267, 1-9.

Böcherer-Linder, K., Eichler, A., \& Vogel, M. (2018) Visualising conditional probabilities-three perspectives on unit squares and tree diagrams. In: Batanero, C., Chernoff, E. (eds) Teaching and Learning Stochastics. ICME-13 Monographs. Springer, Cham. pp. 73-88. 
International Journal of Mathematical, Engineering and Management Sciences

Vol. 5, No. 5, 787-811, 2020

https://doi.org/10.33889/IJMEMS.2020.5.5.062

Carles, M., \& Huerta, M.P. (2007, February). Conditional probability problems and contexts. The diagnostic test context. In Proceedings of the Fifth Congress of the European Society for Research in Mathematics Education, 5(2), 702-710.

Cerdán, F., \& Huerta, M.P. (2007). Problemas ternarios de probabilidad condicional y grafos trinomiales (Ternary problems of conditional probability and trinomial graphs). Educación Matemática, 19(1), 2762 .

Chow, S., \& Ruskey, F. (2003, September). Drawing area-proportional Venn and Euler diagrams. In International Symposium on Graph Drawing (pp. 466-477). Springer, Berlin, Heidelberg.

Chowdhury, M.E., Rahman, T., Khandakar, A., Mazhar, R., Kadir, M.A., Mahbub, Z.B., Islam, K.R., Khan, M.S., Iqbal, A., Al-Emadi, N., \& Reaz, M.B.I. (2020). Can AI help in screening viral and COVID-19 pneumonia?. arXiv preprint arXiv:2003.13145.

Costello, F., \& Watts, P. (2016). People's conditional probability judgments follow probability theory (plus noise). Cognitive Psychology, 89, 106-133.

Da Costa, N.C.A. (1986). Pragmatic probability. Erkenntnis, 25(2), 141-162.

Edo, P., Huerta, M.P., \& Cerdán, F. (2011). Análisis de las resoluciones de problemas de probabilidad condicional mediante grafos (analysis of conditional probability problems using graphs). In: Marín, M., Fernández, G., Blanco, L., \& Paralea, M. (eds) Investigación en Educación Matemática XV (pp. 337-350).

Edwards, A.W.F. (1972). Likelihood: an account of the statistical concept of likelihood and its application to scientific inference. Cambridge and New York: Cambridge University Press.

Falk, R., \& Bar-Hillel, M. (1983). Probabilistic dependence between events. The Two-Year College Mathematics Journal, 14(3), 240-247.

Fawcett, T. (2006). An introduction to ROC analysis. Pattern Recognition Letters, 27(8), 861-874.

Fienberg, S.E., \& Gilbert, J.P. (1970). The geometry of a two by two contingency table. Journal of the American Statistical Association, 65(330), 694-701.

Goldstein, N.D., \& Burstyn, I. (2020). On the importance of early testing even when imperfect in a pandemic such as COVID-19. https://doi.org/10.31219/osf.io/9pz4d.

Good, I.J., \& Mittal, Y. (1987). The amalgamation and geometry of two-by-two contingency tables. The Annals of Statistics, 15(2), 694-711.

Guo, Y.R., Cao, Q.D., Hong, Z.S., Tan, Y.Y., Chen, S.D., Jin, H.J., Tan, K.S., Wang, D.Y., \& Yan, Y. (2020). The origin, transmission and clinical therapies on coronavirus disease 2019 (COVID-19) outbreak-an update on the status. Military Medical Research, 7(1), 1-10.

Hájek, A. (2003). What conditional probability could not be? Synthese, 137(3), 273-323.

Hartigan, J.A., \& Kleiner, B. (1981) Mosaics for contingency tables. In: Eddy W.F. (eds) Computer Science and Statistics: Proceedings of the 13th Symposium on the Interface. Springer, New York. pp. 268-273.

Huerta, M.P. (2009). On conditional probability problem solving research-structures and contexts. International Electronic Journal of Mathematics Education, 4(3), 163-194.

Huerta, M.P. (2014). Researching conditional probability problem solving. In Probabilistic Thinking. Springer, Dordrecht. pp. 613-639. 
International Journal of Mathematical, Engineering and Management Sciences

Vol. 5, No. 5, 787-811, 2020

https://doi.org/10.33889/IJMEMS.2020.5.5.062

Huerta, M.P., Cerdán, F., Lonjedo, M.A., \& Edo, P. (2011). Assessing difficulties of conditional probability problems. In: Pytlak, M., Rowland, T., \& Swoboda, E. (eds) Proceedings of the Seventh Congress of the European Society for Research in Mathematics Education, University of Rzeszów, Poland (pp. 807-817).

Johnson, K.M. (1999). The two by two diagram: a graphical truth table. Journal of Clinical Epidemiology, 52(11), 1073-1082.

Johnson, K.M. (2017). Using Bayes' rule in diagnostic testing: a graphical explanation. Diagnosis, 4(3), 159-167.

Johnson, K.M., \& Johnson, B.K. (2014). Visual presentation of statistical concepts in diagnostic testing: the $2 \times 2$ diagram. American Journal of Roentgenology, 203(1), W14-W20.

Kelly, H., Bull, A., Russo, P., \& McBryde, E.S. (2008). Estimating sensitivity and specificity from positive predictive value, negative predictive value and prevalence: application to surveillance systems for hospital-acquired infections. Journal of Hospital Infection, 69(2), 164-168.

Kent, P., \& Hancock, M.J. (2016). Interpretation of dichotomous outcomes: sensitivity, specificity, likelihood ratios, and pre-test and post-test probability. Journal of Physiotherapy, 62(4), 231-233.

Krämer, W., \& Gigerenzer, G. (2005). How to confuse with statistics or: the use and misuse of conditional probabilities. Statistical Science, 20(3), 223-230.

Lesaffre, E., Speybroeck, N., \& Berkvens, D. (2007). Bayes and diagnostic testing. Veterinary Parasitology, 148(1), 58-61.

Li, B., Yang, J., Zhao, F., Zhi, L., Wang, X., Liu, L., Bi, Z., \& Zhao, Y. (2020). Prevalence and impact of cardiovascular metabolic diseases on COVID-19 in China. Clinical Research in Cardiology, 109, 531538. https://doi.org/10.1007/s00392-020-01626-9.

Li, L., Qin, L., Xu, Z., Yin, Y., Wang, X., Kong, B., Bai, K., Lu, Y., Fang, Z., Song, Q., Cao, K., Liu, D., Wang, G., Xu, Q., Fang, X., Zhang, S., Xia, J., \& Xia, J. (2020). Artificial intelligence distinguishes COVID-19 from community acquired pneumonia on chest CT. Radiology, 200905. https://doi.org/10.1148/radiol.2020200905.

Lipsitch, M., Swerdlow, D.L., \& Finelli, L. (2020). Defining the epidemiology of Covid-19-studies needed. New England Journal of Medicine, 382(13), 1194-1196.

Oldford, R.W., \& Cherry, W.H. (2006). Picturing probability: The poverty of Venn diagrams, the richness of eikosograms. Retrieved from. http://www.stats.uwaterloo.ca/ rwoldfor/papers/venn/eikosograms/paperpdf.pdf.

Parikh, R., Mathai, A., Parikh, S., Sekhar, G.C., \& Thomas, R. (2008). Understanding and using sensitivity, specificity and predictive values. Indian Journal of Ophthalmology, 56(1), 45-50.

Park, M., Cook, A.R., Lim, J.T., Sun, Y., \& Dickens, B.L. (2020). A systematic review of COVID-19 epidemiology based on current evidence. Journal of Clinical Medicine, 9(4), 967.

Peto, J. (2020). Covid-19 mass testing facilities could end the epidemic rapidly. The BMJ (Originally, the British Medical Journal), 368. Available at https://www.bmj.com/content/368/bmj.m1163.long.

Pfannkuch, M., \& Budgett, S. (2017). Reasoning from an eikosogram: an exploratory study. International Journal of Research in Undergraduate Mathematics Education, 3(2), 283-310.

Politzer, G. (2014). Deductive reasoning under uncertainty using a water tank analogy. HAL Archives, Id: ijn_00867284, 1-32.

Powers, D.M. (2011). Evaluation: from precision, recall and F-measure to ROC, informedness, markedness and correlation. Journal of Machine Learning Technologies, 2(1), 37-63. 
International Journal of Mathematical, Engineering and Management Sciences

Vol. 5, No. 5, 787-811, 2020

https://doi.org/10.33889/IJMEMS.2020.5.5.062

Rothan, H.A., \& Byrareddy, S.N. (2020). The epidemiology and pathogenesis of coronavirus disease (COVID-19) outbreak. Journal of Autoimmunity, 102433. https://doi.org/10.1016/j.jaut.2020.102433.

Rushdi, A.M., Rushdi, M.A. (2017). Switching-algebraic analysis of system reliability. In: Ram, M., Davim, J. (eds) Advances in Reliability and System Engineering (pp. 139-161). Management and Industrial Engineering. Springer, Cham. Switzerland.

Rushdi, A.M.A., \& Talmees, F.A. (2018). An exposition of the eight basic measures in diagnostic testing using several pedagogical tools. Journal of Advances in Mathematics and Computer, Science, 26(3), 117.

Rushdi, A.M.A., \& Talmees, F.A. (2019). Computations of the eight basic measures in diagnostic testing. In: Advances in Mathematics and Computer Science. International, Hooghly, West Bengal, India.

Rushdi, R.A., \& Rushdi, A.M. (2018a). Karnaugh-map utility in medical studies: the case of fetal malnutrition. International Journal of Mathematical, Engineering and Management Sciences, 3(3), 220244.

Rushdi, R.A., \& Rushdi, A.M. (2018b). Common fallacies of probability in medical context: a simple mathematical exposition. Journal of Advances in Medicine and Medical Research, 26(1), 1-21.

Rushdi, R.A., Rushdi, A.M., \& Talmees, F.A. (2018). Novel pedagogical methods for conditionalprobability computations in medical disciplines. Journal of Advances in Medicine and Medical Research, 25(10), 1-15.

Rushdi, R.A.M., \& Rushdi, A.M.A. (2019). Mathematics and examples for avoiding common probability fallacies in medical disciplines. In: Current Trends in Medicine and Medical Research. International, Hooghly, West Bengal, India.

Salman, F.M., Abu-Naser, S.S., Alajrami, E., Abu-Nasser, B.S., \& Alashqar, B.A. (2020). COVID-19 detection using artificial intelligence. International Journal of Academic Engineering Research, 4(3), 18-25.

Sethy, P.K., \& Behera, S.K. (2020). Detection of coronavirus disease (COVID-19) based on deep features and support vector machine. International Journal of Mathematical, Engineering and Management Sciences, 5(4), 643-651.

Shindo, T., Takahashi, T., Okamoto, T., \& Kuraishi, T. (2012). Evaluation of diagnostic results by Bayes' theorem. IEEJ Transactions on Electrical and Electronic Engineering, 7(5), 450-453.

World Health Organization (2020). WHO Director-General's opening remarks at the media briefing on COVID-19 on March 11, 2020. Available at https://www.who.int/dg/speeches/detail/who-director-generals-opening-remarks-at-the-media-briefing-on-covid-19---11-march-2020.

Wu, D., Wu, T., Liu, Q., \& Yang, Z. (2020). The SARS-CoV-2 outbreak: what we know. International Journal of Infectious Diseases, 94, 44-48.

Yang, J., Zheng, Y., Gou, X., Pu, K., Chen, Z., Guo, Q., Ji, R., Wang, H., Wang, Y., \& Zhou, Y. (2020). Prevalence of comorbidities in the novel Wuhan coronavirus (COVID-19) infection: a systematic review and meta-analysis. International Journal of Infectious Diseases, 94, 91-95. 